\title{
Erratum: Excitonic collective modes in Weyl semimetals [Phys. Rev. B 98, 165133 (2018)]
}

\author{
N. S. Srivatsa and R. Ganesh
}

(Received 22 June 2020; published 13 July 2020)

DOI: 10.1103/PhysRevB.102.039901

We correct errors in Sec. III of our article which presents a simple model for an interacting Weyl semimetal. We consider an undoped Weyl semimetal with two valleys, labeled " $R$ " and " $L$ ". We define excitonic operators, given by $\hat{\rho}_{\eta \lambda}(\mathbf{q})=$ $\sum_{\mathbf{k}} c_{c, \eta, \mathbf{k}+\mathbf{q}}^{\dagger} c_{v, \lambda, \mathbf{k}}$, where $\eta, \lambda=L / R$ are valley indices. The $c$ and $v$ indices represent the conduction and valence bands, respectively. The bare susceptibility to these orders is given by

$$
\chi_{\alpha \beta, \eta \lambda}(\mathbf{q}, \omega)=\sum_{\mathbf{k}} \frac{\delta_{\alpha \eta} \delta_{\beta \lambda}}{\left(E_{\mathbf{k}+\mathbf{q}}+E_{\mathbf{k}}\right)+\omega+i 0^{+}},
$$

where $E_{\mathbf{p}}=|\mathbf{p}|$ is the quasiparticle energy. The expression in Eq. (11) of the published version has an incorrect minus sign in the denominator. The correct expression can be recovered by defining $\tilde{\omega} \equiv-\omega$. The results in the article regarding $\chi_{\alpha \beta, \eta \lambda}(\mathbf{q}, \omega)$ should be reinterpreted as addressing $\chi_{\alpha \beta, \eta \lambda}(\mathbf{q}, \tilde{\omega})$.

We next consider interaction terms at low energies. In the article, we had considered three processes, all of which elevate a pair of electrons from the valence band into the conduction band. We had represented these processes pictorially in Fig. 2. However, such interactions do not lead to the generalized random-phase approximation (GRPA) form as presented in Eqs. (15) and (16). We correct this discrepancy by considering a different set of interactions, representing the scattering of particle-hole pairs,

$$
\begin{aligned}
\mathcal{H}_{\text {int }}^{\text {simp. }}= & -\frac{\alpha}{N} \sum_{\mathbf{k}, \mathbf{k}^{\prime}, \mathbf{q}} \sum_{\mu=R, L} c_{c, \mathbf{k}+\mathbf{q}, \mu}^{\dagger} c_{v, \mathbf{k}^{\prime}-\mathbf{q}, \mu}^{\dagger} c_{c, \mathbf{k}^{\prime}, \mu} c_{v, \mathbf{k}, \mu}-\frac{\beta}{N} \sum_{\mathbf{k}, \mathbf{k}^{\prime}, \mathbf{q}} c_{c, \mathbf{k}+\mathbf{q}, R}^{\dagger} c_{v, \mathbf{k}^{\prime}-\mathbf{q}, L}^{\dagger} c_{c, \mathbf{k}^{\prime}, L} c_{v, \mathbf{k}, R} \\
& -\frac{\beta}{N} \sum_{\mathbf{k}, \mathbf{k}^{\prime}, \mathbf{q}} c_{c, \mathbf{k}+\mathbf{q}, L}^{\dagger} c_{v, \mathbf{k}^{\prime}-\mathbf{q}, R}^{\dagger} c_{c, \mathbf{k}^{\prime}, R} c_{v, \mathbf{k}, L}-\frac{\gamma}{N} \sum_{\mathbf{k}, \mathbf{k}^{\prime}, \mathbf{q}} c_{c, \mathbf{k}+\mathbf{q}, R}^{\dagger} c_{v, \mathbf{k}^{\prime}-\mathbf{q}, L}^{\dagger} c_{c, \mathbf{k}^{\prime}, R} c_{v, \mathbf{k}, L}-\frac{\gamma}{N} \sum_{\mathbf{k}, \mathbf{k}^{\prime}, \mathbf{q}} c_{c, \mathbf{k}+\mathbf{q}, L}^{\dagger} c_{v, \mathbf{k}^{\prime}-\mathbf{q}, R}^{\dagger} c_{c, \mathbf{k}^{\prime}, L} c_{v, \mathbf{k}, R} .
\end{aligned}
$$

Here, $\alpha, \beta$, and $\gamma$ correspond, respectively, to small-momentum intravalley processes, small-momentum intervalley processes, and large-momentum intervalley processes. We take all three coupling constants to have a factor of $1 / N$, where $N$ is the system size. Such a factor will naturally arise from the Fourier transform of a coupling term that is local in real space. These three processes are shown pictorially in Fig. E1 which replaces Fig. 2 of the published article.

The interaction terms can be written as

$$
\begin{aligned}
\mathcal{H}_{\text {int }} \sim & -\frac{\alpha}{N} \sum_{\mathbf{q}} \sum_{\mu=R, L} \hat{\rho}_{\mu, \mu}(\mathbf{q}) \hat{\rho}_{\mu, \mu}^{\dagger}(\mathbf{q})-\frac{\beta}{N} \sum_{\mathbf{q}}\left\{\hat{\rho}_{R, R}(\mathbf{q}) \hat{\rho}_{L, L}^{\dagger}(\mathbf{q})+\hat{\rho}_{L, L}(\mathbf{q}) \hat{\rho}_{R, R}^{\dagger}(\mathbf{q})\right\} \\
& -\frac{\gamma}{N} \sum_{\mathbf{q}}\left\{\hat{\rho}_{R, L}(\mathbf{q}) \hat{\rho}_{R, L}^{\dagger}(\mathbf{q})+\hat{\rho}_{L, R}(\mathbf{q}) \hat{\rho}_{L, R}^{\dagger}(\mathbf{q})\right\} .
\end{aligned}
$$

(a)

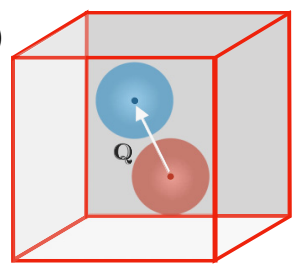

(b)

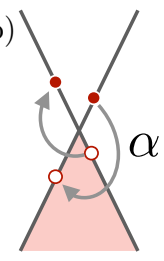

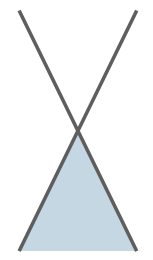

(c)

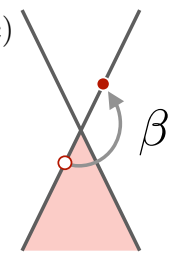

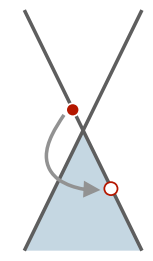

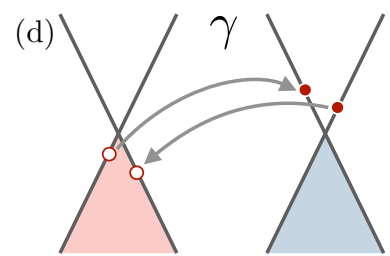

FIG. E1. (a) Brillouin zone for a three-dimensional lattice, assumed to be cubic for simplicity. The spherical regions around Weyl points show linear dispersion. We approximate the Brillouin zone as consisting only of these spherical regions. (b)-(d) Interaction processes at low energies. We have processes with small-momentum transfer that involve the same valley $(\alpha)$ or different valleys $(\beta)$, apart from largemomentum-transfer intervalley scattering $(\gamma)$. 
We quadratically decouple this interaction,

$$
\mathcal{H}_{\text {int }}^{\text {simp }} \rightarrow \frac{1}{N} \sum_{\mathbf{q}}\langle\hat{O}(\mathbf{q})\rangle D^{\text {simp }} \hat{O}^{\dagger}(\mathbf{q})+\text { H.c. },
$$

where $\hat{O}(\mathbf{q})=\left[\hat{\rho}_{L, L}(\mathbf{q}), \hat{\rho}_{R, R}(\mathbf{q}), \hat{\rho}_{L, R}(\mathbf{q}), \hat{\rho}_{R, L}(\mathbf{q})\right]$ and

$$
D^{\text {simp }}=(-)\left[\begin{array}{llll}
\alpha & \beta & 0 & 0 \\
\beta & \alpha & 0 & 0 \\
0 & 0 & \gamma & 0 \\
0 & 0 & 0 & \gamma
\end{array}\right] .
$$

Following the GRPA prescription given in Sec. II, we obtain four collective modes from the condition $\operatorname{det}\left[1+D^{\text {simp }} \chi^{0}(\mathbf{q}, \tilde{\omega})\right]=0$. Here, we have used $\tilde{\omega}$ in place of $\omega$ as described below Eq. (1) above.

Using the form of $\chi$ as given in Eqs. (12) and (13) of the article, we obtain four solutions,

$$
\tilde{\omega}_{i}=q-2 k_{c} \exp \left\{\frac{6\left(q k_{c}-t_{i}\right)}{q^{2}}\right\}, \quad i=1-4,
$$

where $t_{i}$ are given by

$$
t_{1,2}=\frac{\frac{1}{\alpha \pm \beta}-\pi k_{c}^{2}}{2 \pi}, \quad t_{3,4}=\frac{\frac{1}{\gamma}-\pi k_{c}^{2}}{2 \pi} .
$$

To find the form of the binding energy, we consider a simple example with $\gamma>0$ and $\gamma^{-1} \gg \pi k_{c}^{2}$, corresponding to weak interactions. For small $\mathbf{q}$, a collective mode appears with the binding energy given by $E_{b} \approx 2 k_{c} \exp \left(-3 / \pi q^{2} \gamma\right)$. As discussed in the article, this resembles the binding energy of a Cooper pair in a three-dimensional metal.

We note that these corrections do not alter the qualitative statements given in the article. The results and discussions in other sections are correct as published, including Secs. IV and V that discuss a Hubbard model.

We thank M.-F. Yang for pointing out this error. 